\title{
Emergence and Simulation of Bionic Swarm Intelligence Type X Party Material Flow
}

\author{
Hanping Hou and Shoubo Xu \\ School of Economics and Management, Beijing Jiaotong University, Beijing 100044, P.R. \\ China xusb@263.net hphou@sohu.com houhanping@126.com
}

\begin{abstract}
Through the simulation of biological Swarm Intelligence generation, a new kind of material flow structure that can be adapted to complex environments, X Party Material Flow (X-PMF), can be established. According to results from the simulation experiments, the PMF, if in the chaord state during the process of autonomy and adaptation, can realize the emergence of Xparty Swarm Intelligence.
\end{abstract}

Keywords: Emergence, Logistics, Material flow, Simulation, Supply chain, Swarm intelligence (SI), X party material flow (XPMF)

\section{INTRODUCTION}

In traditional logistics theories, it's usual to select from established multi-party logistics under some certain environments (e.g., 1PL-4PL). Viewing from the logistics operation and management process, they are based on the control theory. That's to say, by adopting the method of negative feedback, Top-Down central control and decision-making, logistics operation is applicable to the case in which environmental changes can be predicted. However, under uncertain and complex environments, it'll be difficult to organize and implement such kind of logistics mode based on the traditional control theory [1].

Based on the Material Flow (MF) [2-3], as well as inspired by biological swarm behaviors, the author thinks that through the bionics, a new type of MF mode adapted to complex environments can be possibly established. We can often see the swarm phenomenon of bions (e.g., birds, ants, fishes, etc.), which is favorable for them to find food and escape from predators. They individually possess low intelligence and act simply, but as a swarm show high intelligence and complex behaviors. The characteristics of intelligent behaviors emerging from such kind of low-intelligence or no-intelligence parties through cooperation are called Swarm Intelligence (SI) [4-5]. SI enables bions not only to survive the cruel nature, but also to get highly adaptable to environmental changes. Viewing from the bionics angle, under uncertain and complex environments, the Party of Material Flow (PMF) [6] can be considered as a low-intelligent or no-intelligent bion. Through a bionic study of rules on individual PMF behaviors, emergence mechanism of PMF Swarm Intelligence shall be explored, and finally a new kind of X-PMF [7-8] applicable to complex environments can be established. 


\section{PMF AND ITS CHARACTERS}

On basis of the MF nature [9-10], PMF means any organization or individual capable of independently engaging in MF operation and management. Under uncertain and complex environments, one PMF will behave extremely like a bion. We can further recognize and understand the PMF's characteristics through bionics:

1. Intelligence. Under complex environments, the PMF can neither show very high intelligence, nor have long-run strategic objectives and behavior evolution mechanism. It can respond, to a certain degree, to changes to key factors in exterior environment or behaviors of other parties and it can act according to simple rules.

2. Autonomy. PMF can control its own behavior and internal state and is capable of independently making decisions and taking actions, without the need to receive the command or order from any other PMFs.

3. Mobility. The bion finds food, evades from natural enemies or overpasses obstacles through motion. According to the PMF nature, similarly we can divide PMFs on the supply chain into spatially still or mobile ones.

4. Adaptability. This character means that PMF can continue to interact with the environment and other PMFs, and during such interactions incessantly study or accumulate experiences. By changing their own structures and modes of conduct, they influence and change the environment.

\section{SIMULATION OF X-PMF SWARM INTELLIGENCE (SI)}

The formation of biological SI undoubtedly gives inspiration to establishment of a new type of MF mode adaptable to complex environments. Below let's take the ant swarm seeking food bionically as an example [11], start from the multi-agent simulation and modeling concept [12], study the rules of conduct of PMF under complex environments, and carry out simulation experiments to prove that PMF will cause the emergence of swarm Intelligence of X-PMF in case of simple rules.

\subsection{Model Assumption}

1. Ant means PMF. Under complex environments, the PMF, like low-intelligence ant, possesses the capability of selecting movement paths, conducting information interactions with other ants and adaptability, etc.. The vision range is narrow, for example, the supply chain enterprises may select Class-I and Class-II suppliers. However, it's likely that they know nothing about Class-III suppliers.

2. Obstacles mean objects, which are encountered and should be evaded from during PMF movements, e.g., jam, ban, natural or man-made disasters, etc..

3. Foods means other cooperative parties, targets or objects required by the PMF to meet its own growth, e.g., consumption PMF, owner PMF, supply PMF and other homogeneous or heterogeneous PMFs.

4. Pheromone refers to relevant flags, information, experiences and traces, etc. released by the PMF, which possesses a certain fortune (foods), to the 
environment. Each PMF can only sense the environmental information within its vision range, while the pheromone disappears in the environment at a certain rate.

\subsection{PMF Behavior Rules}

1. Mobility rule. When there is no pheromone around for guidance, the ant will randomly wander around in quest of information on neighboring parties and foods, etc.; in order to prevent taking turns in former places. Good at learning, the ant can remember which points it has recently passed. If sensing that the next scheduled point has been passed, the ant will try to evade from it; each ant will move towards the direction with the most pheromone favorable to itself.

2. Rule for finding food. Each ant will search for food within the perceivable range. The nearest point with foods, if detected, will be chosen; in case of no food, it'll see whether there is pheromone, and comparably see at which point the amount of pheromones is maximal. Thus, it'll approach the place with many pheromones; each ant will mostly make mistakes with little probability. So they don't all move towards the point with the most pheromones, thus preventing local optima.

3. Rule for evading from obstacles. In case of obstacles existing in the direction an ant moves towards, it'll randomly select another direction. Moreover, if there is instruction for pheromone, it'll behave following the rule for finding food.

\subsection{Model Simulation}

Under the abovementioned model assumption, Netlogo open-type simulation software (Programmable modeling environment presented by CCL and ComputerBased Modeling, American Northwestern University) is employed with its internal model library being called. Through setup and adjustment of various condition parameters above, simulation experiments have been conducted on PMF executing simple rules, as shown in Fig. 1 (For purpose of clear display, the snapshot has been processed). The results show that PMF will result in the emergence of X-PMF SI as time evolves.

\subsection{Result Analysis}

1. The party's autonomy and adaptability are two kinds of basic strategies leading to the emergence of SI. During the process of the party's autonomy and adaptability, each party keep its behavior not only under a certain autonomy and flexibility, but also mutually balanced and coordinated, placing the multi-party system between chaos and order, namely the chaord state [1]. At this time, the system gives birth to the most powerful adaptability and innovative capacity, thus showing good SI. 

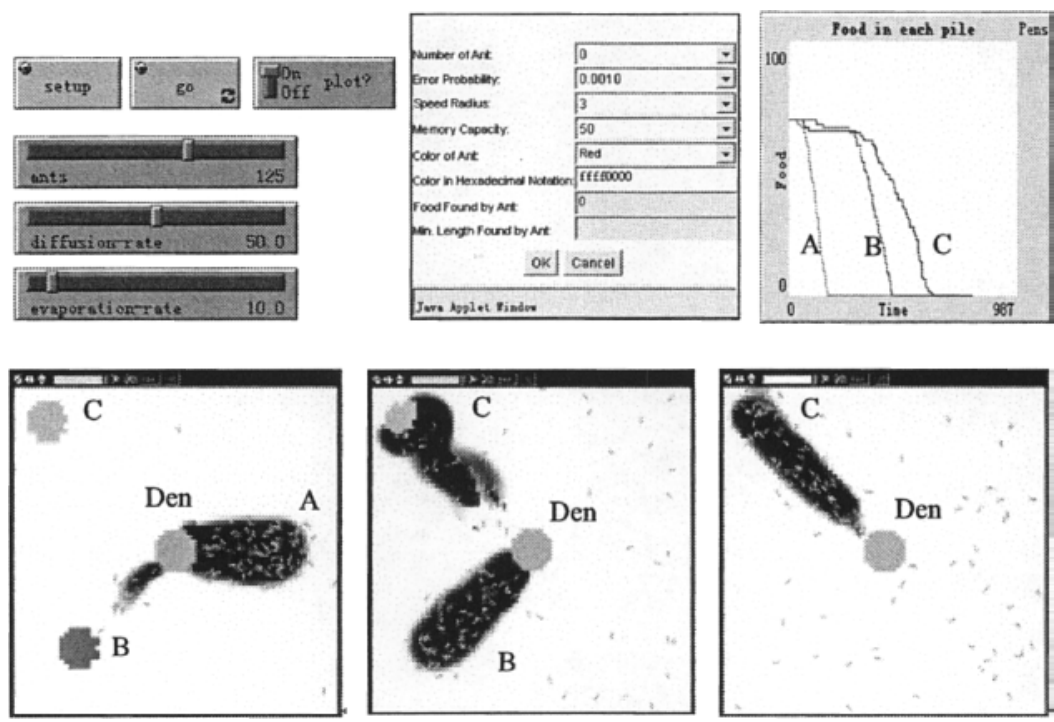

Figure 1. Model Parameter Setup and Simulation Outcome (A, B and C in the figure as the food source)

2. Each party executing the simple rule can produce the emergence of multi-party SI. The solutions to complex environments emphasize the realization of mutual coordination among individuals and SI. It doesn't emphasize how strong the function of every party is. On the contrary, the simpler the party's function is, the better it is. The reason is that simple functions can easily ensure correct and reliable operation as well as relatively easy realization.

3. Bottom-Up organization behaviors. The emergence of ant swarm in the simulation experiment results from Bottom-Up operation process. There is no central controller. The whole ant swarm is organized without an organizer, and parties are coordinated with each other without one coordinator. Therefore, in the future more and more new MF modes will adopt the method of decentralization, replacing the originally centralized and integrally optimized operating system with a separate, self-organized and localized one.

4. Emergence cannot be accurately predicted. In the simulation experiments, due to randomness at movement and small probability error of individuals, as well as indirect interactions through pheromone among individuals, which can make individuals freely access or exit at any time, experiment results indicate random and uncertain changes in quantity, structure and shape of swarm emergence. Therefore, emergence can be observed, but cannot be accurately predicted. Structure designers can only control the rules of party behavior, but cannot design the results of structural emergence. In this case, $\mathrm{X}$, the swarm quantity of multiPMF cannot be determined in advance, and it is called X-PMF. 


\section{CONCLUSIONS}

Through the bionic Swarm Intelligence emergence process, a new kind of MF mode that can be adapted to complex conditions -- X Party Material Flow (X-PMF) -can be constructed. X-PMF is the MF mode featuring Swarm Intelligence emerging with the interaction and mutual adaptation of multiple simple autonomic PMF under no central control. It focuses on the emphasis of autonomy and adaptability of PMF. It can change its own behaviors under interactions with the environment, resulting in the emergence of Swarm Intelligence by X-PMF under the chaord state, so as to reach the reasonable state adapted to complex environments.

\section{REFERENCES}

1. N. Zheng, X. Jia, and Z. Yuan, A survey of controls science and technology (in Chinese), Acta Automatica Sinica. Volume 28, Supplement, pp.7-11, (2002).

2. S. Xu, A new discipline in the era of knowledge economy: Material Flow science and technology, Systems Research and Behavioral Science. Volume 23, Number 2, pp.251$257,(2006)$.

3. S. Xu, On Material Flow (in Chinese), China Business and Market. Volume 19, Number 4, pp.4-7, (2005).

4. S. Hackwood and G. Beni, Self-organization of sensors for swarm intelligence, in Proc. of IEEE International Conference on Robotics and Automation (IEEE Press: Piscataway, NJ, 1992), pp.819-829.

5. J. Kennedy, and R.C. Eberhart, Swarm intelligence (Morgan Kaufmann division of Academic Press: San Francisco, 2001).

6. S. Xu, Scientific classification on Material Flow, Journal of Beijing Jiaotong University (Social Sciences Edition). Volume 4, Number 4, pp.11-15, (2005).

7. H. Hou and S. Xu, Structure and optimization of X Party Material Flow company based on XPMF fractal theory, in Proc. of 2005 International Conference on Management Science \& Engineering $\left(12^{\text {th }}\right)$ (Harbin University of Technology Press: Harbin, 2006), pp.20-21.

8. H. Hou, Theory of X's Party Material Flow, Joumal of Northern Jiaotong University (Social Science Edition). Volume 2, Number 4, pp.25-28, (2003).

9. H. Hou, The nature of Material Flow and logistics dislocation, Group Economy. Number 9, pp.175-176, (2005).

10. Y. Song and H. Hou, The nature of Material Flow and MF management, People Daily. July $20,(2005)$.

11. M. Dorigo and C. Gambardella, Ant colony system: a cooperative learning approach to the traveling salesman problem, IEEE Trans Evolution Compute. Volume 1, Number 1, pp.53$66,(1997)$.

12. W. Li, Modeling tool of agent based simulation, Computer Education. Number 2, pp.47$51,(2005)$.

13. D.W. Hock, Birth of the chaordic age (Locus Publishing Company: 1999). 